\title{
Probabilistic Demand Prediction Model for En-Route Sector
}

\author{
Wen Tian, Ying Zhang, and Yinfeng Li, and Huili Zhang
}

\begin{abstract}
Although airspace congestion is becoming more and more serious with the increase of the air traffic flow, there have been still no mature and effective methods and models developed for measuring the uncertainty of the air traffic flow, so that the air traffic prediction is lack of accuracy. Thus, in this paper we extract the numerical characteristics of the random variables during the flight process, and then establish the probability density functions and en-route sector demand prediction model based on the probability distributions. Through comparing the actual operation data and the prediction data of the aircraft, the variation of the sector traffic flow demand and its probability can be obtained based on the model proposed in the paper. The model in this paper remedies the insufficiency of the traditional flow prediction methods which merely provide static prediction results, and thus can be a useful decision support tool for the air traffic flow managers to dynamically know about the sector traffic demand and its accuracy in the future.
\end{abstract}

Index Terms-Air traffic management, en-route sector demand, probabilistic prediction, uncertianty measuring.

\section{INTRODUCTION}

With the rapid development of the air transportation in China, the air traffic demand is increasing. Due to be lack of mature air traffic demand prediction measuring mechanism and methods, the air traffic management system in China cannot meet the need of the air traffic flow growth. According to such continuously changeable air traffic demand, it is necessary to establish tactic and pre-tactic air traffic demand prediction model and methodology as the basis of the scientific air traffic flow management, which is important for high efficient and safe air transportation in China.

Traditional traffic demand prediction methods contain strategic and tactic prediction. The strategic prediction of the traffic demands includes the method based on linear system theory [1], [2], the intelligent model prediction methods based on knowledge discovery [3], [4], the methods based on nonlinear system theory [5]-[7] and the combinational methods. The tactic prediction of the traffic demands calculates the aircraft count in each time interval through forecasting the trajectory [8]. All the methods above are focusing on the deterministic traffic demand prediction, ignoring the influence of the stochastic flight process to the

Manuscript received November 14, 2014; revised August 22, 2015.

Wen Tian, Ying Zhang, and Yinfeng Li are with College of Civil Aviation, Nanjing University of Aeronautics and Astronautics Nanjing, China (e-mail: tianwen0665@qq.com, yoyozhying@163.com, 1i070320217@126.com).

Huili Zhang is with Central and Southern Regional Air Traffic Management Bureau, Guangzhou Area Control Centre, Guangzhou, China (e-mail: zhl.83@163.net). traffic demand prediction. Thus, the traditional traffic demand prediction methodology cannot match the need of the air traffic flow management in practical operation.

Consequently, numerous efforts are under way to incorporate uncertainty explicitly into air traffic demand prediction these years. Meyn [9] considers that merely depending on the forecast traffic demands utilizing the position of individual aircraft from deterministic perspective cannot realize the overall air traffic demand prediction which is based upon all of the aircraft. According to the uncertain influence of the traditional trajectory prediction to the flow prediction, Sandip [10] points out that the uncertainty and complexity is inherent in the traffic flow prediction, and established the aggregated dynamic stochastic model based on the Poisson distribution. Wanke [11], [12] researches the traffic demand uncertainty in sectors, and analyzes the main factors affecting the demand prediction. Chatterji [13] measures the uncertainty of the sector traffic demand prediction based on the stochastic departure time. Most of these researches don not provide complete measuring models and methods of the sector traffic demand uncertainty, and thus it is difficult to forecast possible changes and their possibilities of the air traffic flow in the sectors at longer look-ahead time (LAT), which causes inaccuracy of the present-day traffic flow prediction. Consequently, in order to provide more practical strategies for the decision support of the air traffic flow management, it is necessary to establish probabilistic sector demand prediction model to measure the uncertainty of the traffic demand in the sectors at LAT.

In this paper, according to the stochastic characteristics of the aircraft flight process in the sectors, we analyze the sector entry time, flight time and exit time of the aircraft, establish the probabilistic sector demand prediction model, and exact the numerical characteristics' of the random variables of the aircraft during the flight process based on the real flight data and prediction data. Through combining such characteristics with the model, we obtain the probabilistic distribution and its variation rules of the sector air traffic demand at certain LAT.

\section{PROBLEM DESCRIPTION}

For the sake of convenience, the 4 dimensional spaces (three dimension space, one dimension time) is simplified into 2 dimensional network model, including airspace, airports, route points, sectors and routes. The whole airspace is divided into two kinds of airspace: objective airspace and non-objective airspace. The airspace containing objective airports is the objective airspace, and is composed of several sectors. The airspace containing the other airports is the 
non-objective airspace as a whole. An aircraft takes off from the departure airport, passing by the route points, entering and exiting the sectors, finally landing on the objective airport. The whole flight process represented by solid lines is approximated as the straight flight from one point to the next, while the real flight process of the aircraft trajectory is represented by dashed lines in the figure.

In the practical operation, traffic demand uncertainties arise from many sources: flight cancellations, departure time changes, and initiation of previously unscheduled flights, uncertainties in wind forecasting and aircraft performance modeling, and unforeseen changes in flight route and cruising altitude due to weather and air traffic control intervention. Such factors above cause the aircraft real flight trajectory deviating from the ideal straight line flight from one point to the next, which initiating the flight time and position of the aircraft are unpredictable. Such unpredictability means that the sector entry time and exit time of the aircraft is stochastic, the sector occupied time of the aircraft is probabilistic, and such time follows a certain probabilistic distribution (Fig. 1). Therefore, the predicted airspace traffic demand based upon sector entry, exit and occupied time is probabilistic.

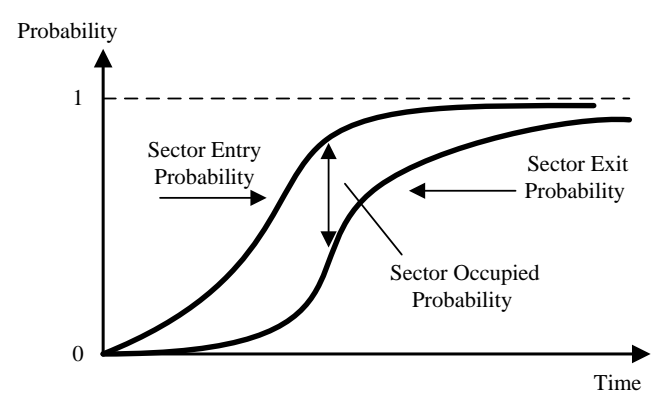

Fig. 1. Probability of sector entry \& exit \& occupied airspace.

In this paper, through analyzing the stochastic characteristics of three main factors-sector entry, exit and occupied time, we establish the probabilistic sector demand prediction model. Based upon the practical operation data and prediction data, we calculate the numerical characteristics of several random variables, and obtain the sector traffic demand probabilistic distribution and its variation rules at certain LAT.

\section{MATHEMATIC MODEL}

\section{A. Parameters}

- $\xi$ is the output of the experiment $\Omega$;

- $T$ is the objective time interval of the traffic demand prediction;

- $F$ is the set of aircraft, aircraft $f_{i} \in F, i=1, \ldots, N, N$ is the total count of all the aircraft;

- $A$ is the set of airports outside the objective airspace, airport $a_{i} \in A, i=1, \ldots, N_{A}, N_{A}$ is the total count of such airports;

- $B$ is the set of airports inside the objective airspace, airport $b_{i} \in B, i=1, \ldots, N_{B}, N_{B}$ is the total count of such airports;

- $C$ is the set of route points inside the objective airspace, point $c_{i} \in C, i=1, \ldots, N_{C}, N_{C}$ is the total count of such points, $A \subset C, B \subset C$;

- $d(k, l)$ is the distance between to points $(k, l \in C)$, and $d(k, l)=d(l, k)$;

- $t_{k, i}^{a t a}$ is the actual arrival time of the aircraft $f_{i}$ to the point $k, t_{k, i}^{e t a}$ is the estimated arrival time of the aircraft $f_{i}$ to the point $k, d_{k, i}^{a}$ is the delay time of the aircraft $f_{i}$ to the point $k$, and $t_{k, i}^{a t a}=t_{k, i}^{e t a}+d_{k, i}^{a}, k \in C, f_{i} \in F$ $t_{k, i}^{a t a}, t_{k, i}^{e t a} \in T$

- $t_{k, i}^{\text {atd }}$ is the actual departure time of the aircraft $f_{i}$ from the point $k, t_{k, i}^{\text {etd }}$ is the estimated departure time of the aircraft $f_{i}$ from the point $k, d_{k, i}^{d}$ is the delay time of the aircraft $f_{i}$ from the point $k$, and $t_{k, i}^{\text {atd }}=t_{k, i}^{\text {etd }}+d_{k, i}^{d}, k \in C$, $f_{i} \in F, t_{k, i}^{a t d}, t_{k, i}^{e t d} \in T$

- $t_{i}^{(k, l)}$ is the flight time of the aircraft $f_{i}$ from the point $k$ to $l$, and $t_{i}^{k, l}=t_{i}^{l, k}, k \neq l, k, l \in C, f_{i} \in F$;

- $\quad \underline{v}$ is the flight speed vector of an aircraft;

- $p_{I N, i}$ is the probability of aircraft $f_{i}$ entering into a sector at time $t, p_{O U T, i}$ is the probability of aircraft $f_{i}$ exiting from a sector at time $t, p_{i}$ is the probability of aircraft $f_{i}$ occupying a sector at time $t$, and $p_{i}=p_{I N, i}-p_{\text {OUT }, i}$;

- $\quad P_{N}[n]$ is the probability that there are $n$, aircraft existing in the sector in some time interval.

\section{B. Stochastic Character during the Flight}

1) Stochastic character of arrival time

$t_{k, i}^{a t a}=t_{k, i}^{e t a}+d_{k, i}^{a}$ is the actual arrival time of the aircraft $f_{i}$ to the point $k$. Because $t_{k, i}^{\text {eta }}$ is scheduled in advanced, is a deterministic variable. Because of some factors above, $d_{k, i}^{a}$ is probabilistic, and $t_{k, i}^{a t a}$ is a random variable. $\Omega$ is a "sample space", $\xi$ is the probabilistic event "aircraft $f_{i}$ arriving to $k^{\prime \prime}$, and $d_{k, i}^{a}=d_{k, i}^{a}(\xi)$.

According to Law of large numbers: If the experiment $\Omega$ is repeated $n$ times and the event occurs $n_{a}$ times, then, with $a$ high degree of certainty, the relative frequency $n_{a} / n$ of the occurrence of a is close to $\operatorname{Pr}(a) \cong n_{a} / n$. For the given delay time $x$, the probabilistic distribution function of $t_{k, i}^{a t a}$ is $F(x)=\operatorname{Pr}\left\{d_{k, i}^{a}(\xi) \leq x\right\} \cong n(x) / n$, where $n$ is the total times of aircraft $f_{i}$ arriving to $k, n(x)$ is the total times of $d_{k, i}^{a}(\xi) \leq x$.

For the given delay time $x$, the probability density 
function of $t_{k, i}^{a t a}$ is $f(x) \Delta x \cong \Delta n(x) / n$, where $n$ is the total times of aircraft $f_{i}$ leaving from $k, \Delta n(x)$ is the total times of $\left(x \leq d_{k, i}^{a}(\xi) \leq x+\Delta x\right)$.

\section{2) Stochastic character of departure time}

Similarly, for the actual departure time $t_{k, i}^{\text {atd }}$ of aircraft $f_{i}$ from $k, t_{k, i}^{\text {atd }}=t_{k, i}^{e t d}+d_{k, i}^{d}$, where $t_{k, i}^{\text {etd }}$ is a deterministic variable, $d_{k, i}^{d}$ is a random variable. The probability density function of $t_{k, i}^{\text {atd }}$ is $f(y) \Delta y \cong \Delta n(y) / n$, where $n$ is the total times of aircraft $f_{i}$ arriving to $k, \Delta n(y)$ is the total times of $\left(y \leq d_{k, i}^{d}(\xi) \leq y+\Delta y\right)$.

\section{3) Stochastic character of flight time}

$\Omega$ is a "sample space", $\xi$ is the probabilistic event "aircraft $f_{i}$ flying from $k$ to $l$ ". During the process of the flight from $k$ to $l$, the speed is uncertain, and $\underline{v}=v(\xi)$. For the sake of continence, we simplify the speed as a two dimensional real random variable on the Cartesian axes (Fig. 2 ), where $v_{\tau}$ and $v_{n}$ is respectively the speed component on $\tau$ and $v$ axes.

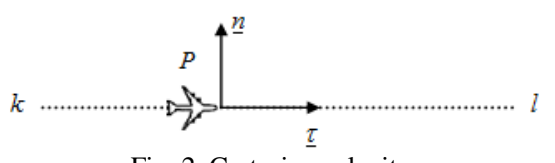

Fig. 2. Cartesian velocity.

Because it is difficult to obtain the real-time aircraft flight data, observing $t_{i}^{(k, l)}$ is easier than $v_{\tau}$, then we convert the stochastic of the speed from $k$ to $l$ into the stochastic of the flight time $t_{i}^{(k, l)}$. For a given flight process with distance $d(k, l)$, the random variable $t_{i}^{(k, l)}$ is equal to $t_{i}^{(k, l)}(\xi)$, and the mean of the random variable $v_{\tau}$ is $\bar{v}_{\tau}(\xi)=d(k, l) / t_{i}^{(k, l)}(\xi)$ (Fig. 3). Observing the $\bar{v}_{\tau} n$ times, the random variable $v_{\tau}$ can be obtained through the random variable $t_{i}^{(k, l)}$ and function $g\left(t_{i}^{(k, l)}\right)$. If $v_{\tau}$ and $\bar{v}_{\tau}$ equivalent, then $v_{\tau}=g\left(t_{i}^{(k, l)}\right)=d(k, l) / t_{i}^{(k, l)}$.

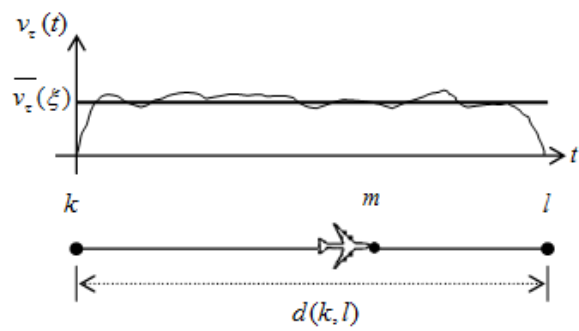

Fig. 3. $\bar{v}_{\tau}(\xi) \&$ Distance for a given journey.

Theorem: $v_{\tau}$ and $\bar{v}_{\tau}$ is equivalent.

Proof: Let $v_{\tau}$ be a real number and let $z$ be a Bernoulli random variable such as:

$$
z\left(v_{\tau}\right)=\left\{\begin{array}{cc}
1 & \text { if } v_{\tau} \leq v_{\tau}<v_{\tau}+d v_{\tau} \\
0 & \text { else }
\end{array},\right.
$$

The expected value of the Bernoulli random variable $z$ is computed:

$$
E\left\{z\left(v_{\tau}\right)\right\}=1 \cdot \operatorname{Pr}\left\{z\left(v_{\tau}\right)=1\right\}=\operatorname{Pr}\left\{v_{\tau} \leq v_{\tau}<v_{\tau}+d v_{\tau}\right\}
$$

Clearly if $v_{\tau}$ is ergodic then $z$ is also ergodic. The temporal mean of $z$ is equal to the expected value of $z E\left\{z\left(v_{\tau}\right)\right\}=\bar{z}\left(v_{\tau}\right)$.

Thus, the following equivalence is given:

$$
\bar{z}\left(v_{\tau}\right)=\operatorname{Pr}\left\{v_{\tau} \leq v_{\tau}<v_{\tau}+d v_{\tau}\right\} \text {, then } \bar{v}_{\tau}=v_{\tau} .
$$

Consequently, the time of aircraft $f_{i}$ flying from $m$ to $l$ is

$$
t_{i}^{(m, l)}=d(m, l) / v_{\tau}=t_{i}^{(k, l)} \cdot d(m, l) / d(k, l)
$$

The arrival time of aircraft $f_{i}$ to $l$ is

$$
t_{l, i}^{a t a}=t_{m, i}^{a t d}+t_{i}^{(m, l)}=t_{m, i}^{a t d}+t_{i}^{(k, l)} \cdot d(m, l) / d(k, l)
$$

Through variable substitution, the probability density function of the arrival time from $k$ to $l$ passing by $m$ is

$$
f_{t_{l, i}^{a(a}}\left(t_{l, i}^{a t a}\right)=\frac{|d(k, l)|}{|d(m, l)|} f_{t_{i}^{(k, l)}}\left(\left(t_{i}^{(m, l)}-t_{m, i_{i}}^{a t d}\right) \frac{d(k, l)}{d(m, l)}\right)
$$

\section{Probabilistic Sector Demand Prediction Model}

If the radar data is sufficient, the probability of aircraft $f_{i}$ passing by point $m$ into the sector at time $t$ is

$$
p_{I N, i}=\int_{-\infty}^{t} f\left(t_{m, i}^{a t a}\right) d t_{m, i}^{a t a}
$$

where $f\left(t_{m, i}^{a t a}\right)$ is the probability density function of the arrival time of the aircraft $f_{i}$ passing by point $m$ into the sector at time $t$. And the probability of aircraft $f_{i}$ passing by point $n$ out of the sector at time $t$ is

$$
p_{\text {OUT }, i}=\int_{-\infty}^{t} f\left(t_{n, i}^{a t a}\right) d t_{l, i}^{a t a}
$$

where $f\left(t_{n, i}^{a t a}\right)$ is the probability density function of the arrival time of the aircraft $f_{i}$ passing by point $n$ out of the sector at time $t$.

If the radar data is insufficient to provide the entry and exit time of aircraft, the (1) (2) are still effective but $f\left(t_{n, i}^{a t a}\right)$ is the probability density function of the arrival time of the aircraft $f_{i}$ flying from $k$ to $n$ with $m$ passed by 
(Fig. 4).

$$
f_{t_{n, i}^{a t a}}\left(t_{n, i}^{a t a}\right)=\frac{|d(k, n)|}{|d(m, n)|} f_{t_{i}^{(k, n)}}\left(\left(t_{i}^{(m, n)}-t_{m, i}^{a t d}\right) \frac{d(k, n)}{d(m, n)}\right)
$$

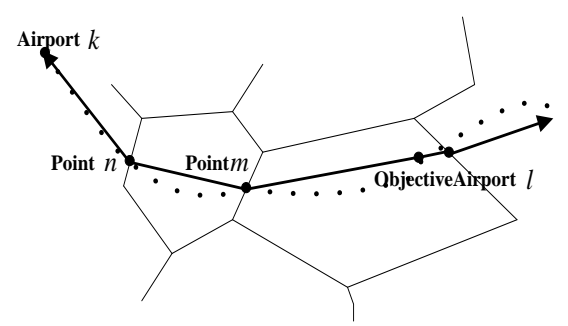

Fig. 4. Process of aircraft flights.

Consequently, the probability of aircraft $f_{i}$ occupying a sector at time $t$ is $p_{i}=p_{I N, i}-p_{O U T, i}$. If in the future time there will be possibly $N$ aircraft flying in a sector, the probability of existing $N$ aircraft in this sector is

$$
\begin{aligned}
& P_{N}[n](0 \leq n \leq N), \text { and } \\
& P_{0}[0]=1, \text { For } i=1 \text { to } N: \\
& \left\{P_{i}[0]=\left(1-p_{i}\right) \cdot P_{i-1}[0], P_{i}[i]=p_{i} \cdot P_{i-1}[i-1],\right. \\
& \text { For } \left.k=1 \text { to }(i-1): P_{i}[k]=p_{i} \cdot P_{i-1}[k-1]+\left(1-p_{i}\right) \cdot P_{i-1}[k]\right\}
\end{aligned}
$$

\section{CASE STUDY}

\section{A. Data Statistic}

According to the operation data of Guangzhou area in the $4^{\text {th }}$ week of Oct. 2009, there are 3946 flights flying into and out of sector AC05 during the whole day. The delay time of every route point submitted to normal distribution, the mean $\mu$ and deviation $\sigma$ are as follows:

TABLE I: PARAMETERS OF SECTOR ENTRY DELAY TIME
\begin{tabular}{|c|c|c|}
\hline Rout Point & $\mu(\mathbf{m i n})$ & $\sigma(\mathbf{m i n})$ \\
\hline BEKOL & 20.1 & 13.3 \\
\hline BIGRO & 13.2 & 11.9 \\
\hline BOKAT & 19.7 & 14.1 \\
\hline CON & 15.6 & 13 \\
\hline GYA & 15.1 & 13.5 \\
\hline IDUMA & 16.1 & 13.5 \\
\hline NOMAR & 20.9 & 14.4 \\
\hline P269 & 13.7 & 12.4 \\
\hline POU & 15 & 8.8 \\
\hline SAREX & 13.5 & 12.2 \\
\hline SHL & 19 & 18.7 \\
\hline TAMOT & 16.9 & 10.9 \\
\hline VIBOS & 22.1 & 13.8 \\
\hline VIPAP & 16.6 & 14.5 \\
\hline
\end{tabular}

\section{B. Experiment Result and Analysis}

According to the probabilistic sector demand prediction model and the delay time parameters and operation data of 3946 flights at 9:00-11:00 on 10/25/2009, the probabilistic demand distribution of sector AC05 can be calculated. In Fig. 5, the aircraft count and its changing rules with different time and different MAPs at a certain LAT (30min) shows that at the same time, with the increase of MAP, the probability corresponding to the count of the aircraft is decreased (for example, the probability of aircraft count $\geq 1$ at $10: 00$ is $99 \%, \geq 2$ is $99 \%, \geq 3$ is $98 \%, \geq 4$ is $97 \%, \geq 5$ is $92 \%, \geq 6$ is $82 \%$ ). That is to say, the count of the aircraft in a sector at the same is impossibly increasing unlimitedly. With the prediction count increasing, the corresponding possibility may decrease. For the same MAP, the probability corresponding to some aircraft counts in the sector at different time will be increasing with time increasing, and decreasing down after the peak value with time increasing (for example, the probability of aircraft count $\geq 5$ is $0.1 \%$ at $9: 30,10.0 \%$ at $10: 00,80.2 \%$ at $10: 30,61.6 \%$ at $11: 00)$. That is to say, with the prediction time far away from present time, the aircraft count and its probability will be at peak at 10:30, then decrease down. Thus, MAP and prediction time are both important factors affecting the sector traffic demand prediction.

TABLE II: PARAMETERS OF SECTOR EXIT DELAY TIME

\begin{tabular}{|c|c|c|}
\hline Rout Point & $\mu(\mathbf{m i n})$ & $\sigma(\mathbf{m i n})$ \\
\hline BIGRO & 17.9 & 14 \\
\hline GURIN & 13.6 & 12.1 \\
\hline GYA & 16 & 12.8 \\
\hline IDUMA & 17.6 & 10.7 \\
\hline LMN & 12.7 & 12.6 \\
\hline POU & 6.9 & 6.2 \\
\hline SAREX & 13.8 & 10.5 \\
\hline SHL & 22.2 & 13.1 \\
\hline SIERA & 21.8 & 13.2 \\
\hline
\end{tabular}

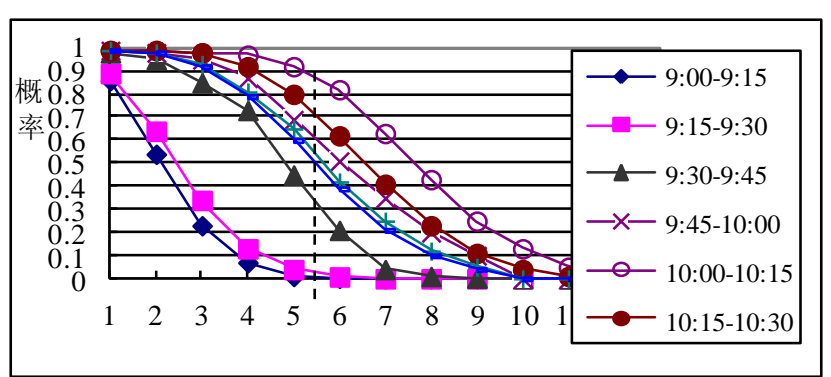

Fig. 5. Probability distribution (LAT=30min).

The probability distribution of aircraft count at 10:00 in sector AC05 at different LATs is in Table II. For the same MAP, with the increase of the LAT, the probability that aircraft count is more than MAP is more and more. Thus, LAT is also relative for the sector traffic demand prediction.

TABLE III: PROBABILISTIC DISTRIBUTION OF DEMANDS AT LATS
\begin{tabular}{|c|c|c|c|}
\hline & LAT $=30$ min & LAT $=\mathbf{6 0 m i n}$ & LAT=90min \\
\hline MAP $=1$ & 0.97521775 & 0.975275486 & 0.975275566 \\
\hline MAP $=2$ & 0.87278898 & 0.873023346 & 0.87302367 \\
\hline MAP $=3$ & 0.674334234 & 0.674779422 & 0.674780036 \\
\hline MAP $=4$ & 0.434183483 & 0.434710686 & 0.434711413 \\
\hline MAP $=5$ & 0.230161697 & 0.230599165 & 0.230599767 \\
\hline
\end{tabular}

\section{CONCLUSIONS}

According to the stochastic characteristics during the aircraft flight process in the sector, we exact the main factors effecting the demand prediction, analyze the sector entry time, exit time and flight time, and establish the probabilistic sector traffic demand prediction model. Based upon the practical operation data, we calculate the numerical characteristics of the random variables during the flight process. Then, with the probabilistic sector demand prediction model, we obtain the probability distribution and its variation rules, and measure the uncertainty of the air traffic demand prediction. In the future research, we hope to 
analyze the influence of the different uncertain factors during the aircraft flight process from more microscopic perspective to realize the uncertainty measuring based upon the more complete and reliable data.

\section{ACKNOWLEDGMENT}

Project supported by the National Natural Science Foundation of China (Grant No. 71301074 )

\section{REFERENCES}

[1] Z. Yang and Z. Zhu, "A real time traffic volume prediction model based on the kalman filtering theory," China Journal of Highway and Transport, vol. 12, no. 3, pp. 63-67, July 1999.

[2] S. Dai and X. Zhou, "Application of kalman filtering theory on prediction of short-term traffic volume," Journal of Harbin University of Commerce (Sciences Edition), vol. 21, no. 6, pp.728-730, Dec. 2005.

[3] X. Zhang and G. He, "The combined forecasting approach based on nonparametric regression for short-term traffic flow of roads with parking spaces," Systems Engineering, vol, 24, no. 12, pp. 21-25, Dec. 2006.

[4] T. Cheng and D. Cui, "Peng data mining for air traffic flow forecasting: A hybrid model of neural-network and statistical analysis," IEEE, July 2003.

[5] X. Bai, X. Ye, and H. Jiang, "Network traffic predicting based on wavelet transform and autoregressive model," Computer Science, vol. 34, no. 7 , pp.47-54, 2007.

[6] D. Huang, J. Song, D. Wang, and J. Cao, "Forecasting model of traffic flow based on arma and wavelet transform," Computer Engineering and Applications, vol. 36, pp. 191-224, 2006.

[7] F. Yang, and S. Zhu, "Short-term traffic flow forecasting based wavele," Journal of Chongqing Jiaotong University, vol. 25, no. 3, pp. 99-102, June 2006.

[8] Y. Peng, M. Hu, and Y. Zhang," Conjecture method of dynamic flying track," Journal of Traffic and Transportation Engineering, vol. 5, no. 1, pp. 62-65, March 2005.

[9] M. Larry, "Probabilistic methods for air traffic demand forecasting [C]//AIAA 2000-4766," AIAA Guidance, Navigation, and Control Conference and Exhibit. Monterey, CA, USA: AIAA, 2002, vol. 5-8.

[10] S. Roy, B. Sridhar, and G. C. Verghese, "An aggregate dynamic stochastic model for air traffic control," presented at 5th USA/Europe ATM 2003 R\&D Seminar, Budapest, Hungary, 2004.

[11] C. Wanke, M. Callaham, and D. Greenbaum, and A. masalonis, "Measuring uncertainty in airspace demand predictions for traffic flow management applications," in Proc. Navigation, and Control Conference and Exhibit, Austin, TX: AIAA, 2003, vol. 11-14.
[12] C. Wanke, S. Mulgund, D. Greenbaum, and L. Song. "Modeling traffic prediction uncertainty for traffic management decision support," in Proc. Navigation, and Control Conference and Exhibit, Providence, RI: AIAA, 2004, vol. 16-19.

[13] G.Chatterji, D. Kim, D. Mulfinger, B. Shridar, and K. Sheth, "Methods for establishing confidence bounds on sector demand forecasts," in Proc. Navigation and Control Conference, Providence, RI: AIAA, 2004.

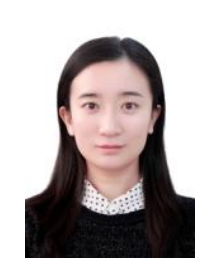

Wen Tian received a bachelor of engineering in electronic information engineering in 2003 from Xidian University, and a Ph.D. in transportation planning and management in 2012 from Nanjing University of Aeronautics and Astronautics. She has been working as a lecturer at Nanjing University of Aeronautics and Astronautics, Nanjing, Jiangsu, China since 2012. Her research interest includes decision support for air traffic management, airspace congestion management and uncertain traffic flow management.

Ying Zhang received a bachelor of engineering in transportation management in 1999, a master in transportation planning and management in 2012, and a Ph.D. in transportation planning and management in 2015, all from Nanjing University of Aeronautics and Astronautics. She has been working as a lecturer at Nanjing University of Aeronautics and Astronautics, Nanjing, Jiangsu, China since 2003. Her research interests include collaborative air traffic flow management, and intelligent air transportation system.

Yinfeng $\mathbf{L i}$ received a bachelor of engineering in transportation management and electronic commerce in 2007, a master in transportation planning and management in 2010, and a Ph.D. in transportation planning and management in 2015, all from Nanjing University of Aeronautics and Astronautics. He has been working as a researcher at National Key Laboratory of Air Traffic Flow Management, Nanjing University of Aeronautics and Astronautics, Nanjing, Jiangsu, China since 2015. Her research interests include airspace management and operation evaluation.

Huili Zhang received a bachelor of engineering in computer application technology in 2001, a master in transportation planning and management in 2007, both from Nanjing University of Aeronautics and Astronautics. She has been working as an air traffic controller at Central and Southern Regional Air Traffic Management Bureau, Guangzhou, China since 2007. Her research interests include air traffic flow management, and risk management. 